\title{
Aesthetic Management in Maxillofacial Prosthesis- A Review
}

\author{
Dr. Nabaprakash Sahu MDS ${ }^{1 *}$, Dr. Amit Shivakant Vathare, MDS ${ }^{2}$, Dr. Rahul Vinay Chandra Tiwari, FOGS, MDS ${ }^{3}$, Dr. \\ Sunil kumar, Senior Lecturer ${ }^{4}$, Dr. Shweta Meshram ${ }^{5}$, Dr. Heena Tiwari, BDS, PGDHHM ${ }^{6}$ \\ ${ }^{1}$ Prosthodontics, Crown \& Bridges, Reader, Department Of Prosthodontics, Hi-Tech Dental College \& Hospital, Health Park, Pandara, Rasulgarh, \\ Bhubaneswar, Odisha India \\ ${ }^{2}$ Consultant Prosthodontist \& Implantologist, Jaysingpur, Shirol, Kolhapur, Maharashtra India \\ ${ }^{3}$ Consultant Oral \& Maxillofacial Surgeon, CLOVE Dental \& OMNI Hospitals, Visakhapatnam, Andhra Pradesh, India \\ ${ }^{4}$ Oral and maxillofacial Surgery, SGT University, Gurugram, Badli, Jhajjar, Haryana India \\ ${ }^{5}$ Consultant Pedodontics and Preventive Dentistry Advanced Dental Hospital, Nagpur, Maharashtra India \\ ${ }^{6}$ Government Dental Surgeon, Chhattisgarh, India
}

DOI: $10.36348 /$ sjm.2020.v05i02.010 $\quad$ | Received: 04.02 .2020 | Accepted: 15.02 .2020 | Published: 22.02 .2020

*Corresponding author: Dr. Nabaprakash Sahu MDS

\section{Abstract}

Facial perception is the first aspect to recognize and understand a person. Thereby, for these reasons, the face has always been significant historically. Moreover, mouth significantly affects the appearance of face. Several people regularly make serious requests to the dentists in order to meet their aesthetic demands, like some of the people pay less attention to the health of their posterior teeth, but they do give attention on the anterior tooth decay, or their malformation and want them to be treated as early as possible. Since the facial region contributes to the self esteem and self respect of an individual both positively and negatively which leads to the prime motivation towards having a beautiful and attractive face. A pleasant face makes people to feel better and also impart positive effects on the social relationships.

Keywords: Aesthetics, maxillofacial defects, maxillofacial prosthesis.

Copyright @ 2020: This is an open-access article distributed under the terms of the Creative Commons Attribution license which permits unrestricted use, distribution, and reproduction in any medium for non-commercial use (NonCommercial, or CC-BY-NC) provided the original author and source are credited.

\section{INTRODUCTION}

The fundamental of a healthy psychological structure lies in an aesthetically acceptable appearance. Human beings always try to maintain equilibrium between his physical and psychological parts. Henceforth, one of the most important duties in this regard belongs to dentists and here appears the importance of the practice of aesthetic dentistry in human life[1]. Maxillofacial prostheses have a special place in the dentistry as science and art of the anatomical, functional as well as cosmetic restoration of any region of maxilla, mandible, or any other region which has a defect due to various causes like trauma, surgical operation, congenital defect, pathology or other reasons. Although the materials and techniques have been widely developed in the recent centuries but the first primitive maxillofacial prostheses were produced much earlier [2]. The dentistry has contributed to the development of an acrylic ocular organ is the production of ocular prostheses by measuring the sockets rather than using traditionally applied methods. During and after the First World War, in the early 20th century, the cooperation of surgeons and dentists began to fabricate the prostheses. Since the restoration of the stomatognathic system with artificial materials with acceptable aesthetic results are very important for the social adaptation and life quality, the maxillofacial prostheses hold a very important place between all the classes of prosthetics[3].

Auricular Prostheses- In order to achieve a natural or almost natural appearance, it is essential to have an appropriate prosthetic reconstruction by using suitable material, for example, auricular defects may be reconstructed by using autogenous tissues [4]. Furthermore, it does not require any additional surgical operation in order to achieve retention and cohesiveness, except a first surgery for the preparation of defect region. In order to prepare an ear prosthesis prototype and also to achieve the mirror image of the ear, the use of optic systems, CT, KIBT and laser surface scanners, CNC, CAD and fast prototyping techniques is very helpful. CT data are also very useful for correct positioning of prosthesis. It is aesthetically important to create a completely adapted frontal line for the auricular prosthesis. It may lead to confusion in cases of mimics, head posture, mandible movements, facial asymmetry, and finally aesthetic. While those problems can be generally solved by randomly digging the frontal border of a master model, desired results may not be always achieved because this 
implementation is not a controlled method [5]. Distance occurring on the frontal border due to movements of head and chin can be solved by providing a barrier on the frontal region of master model and also by elastically preparing the frontal border of prosthesis. However, the digging of the model should be preceded by a clinical evaluation of the soft tissues.

Nasal Prostheses- The execution of the nasal prostheses can be started 4-6 months after the surgery. In order to achieve a success in prosthesis, the shape and size of the defect are very important. Prostheses are more successful in the cases which are protected by the nasolabial sulcus. In the nasal defects, since the lower border tissues are mobile, thereby the prostheses must be prepared as thin and elastic as possible in those areas. The prime factors which affect the aesthetic success of prostheses are masking demarcation lines, suitable creation of contours and compliance of prosthesis surface and skin [6]. The width of nasal wings has to be prepared in such a manner as not to exceed the distance between the inner edges of eyes. Moreover, the conjunction of columella and the skin must be finished as perpendicular and narrow so the demarcation line will be less visible due to shade of the nasal edge. In case of male patients, this region can also be masked by including a mustache. In order to mask the demarcation lines in the lateral and upper regions as well as for retention purposes, the eyeglasses are used. The painting must be performed slightly more markedly than the near skin because of the color loss during painting and finishing phases. After final controls and corrections on the patient, the finishing stage begins [7].

\section{Orbital Prostheses}

Orbital or oculo-facial prostheses comprised of prostheses made for the inner and outer canthi, restoration of upper and lower eyelids and tissues extending from the orbita through the face, except eyeball. Since eye contact is generally consideration in communication between people and therefore for the successful aesthetic, it is very important to consider even minimal differences in the position of eyes, the contour of the eyelids and even minimal differences in the colors of the prosthesis can be discerned easily. It is of great important to take care of the orbital prostheses by masking the borders and artificiality by preparing it in accordance with the color and structure of healthy tissues. Apart from the skin properties, symmetry of the synthetic eye lashes and eyebrows in terms of thickness, color and shape is also very important for aesthetic success. Also, it should be considered to prepare the prosthesis with borders as thin as possible, in such a way that it does not break the harmony of the mimicry [8]. The thick eyeglass with light colored lenses is the most frequently used camouflage method for the borders of these prostheses. The lines and rugae around the eye are exploited in old patients. With the continuous developments in the digital technology, without measuring the face manually, the measurements of the facial region are performed by the fast prototyping data acquired from the laser surface scanners and optical systems by using CAD\&CAM, leading to creation of a model [9]. Since this method records the tissues in a static state, therefore the adaptation in conjunction points can be broken with the movements, however, the movements can be limited with pain. In order to achieve an aesthetic success, the placement of the ocular part of orbital prosthesis should be at the same level as the healthy eye in terms of vertical, horizontal and sagittal axes. Various computerized monitoring techniques can be used for determining the correct eyelid and ocular position, however, for this purpose; a digital image of the patient is created by a digital camera from the lens-object distance and mirror [10]. The retention can be provided by using the tissue undercuts, adhesives, eyeglasses and implants for most cases.

\section{Mid-facial Defects}

The reconstruction of complex facial defects resulting from tissue loss due to trauma, tumors, congenital defects etc should satisfy both the functional as well as aesthetic requirements [11]. Generally there is requirement of very large tissue resection for the treatment of advanced tumors in the middle facial region. The loss of both extra oral and intra oral tissues occurs in such defects. Upper lip, cheek, nasal, or orbital structures may be included in that lost of tissue. Furthermore, soft tissues, mandible, teeth and segments of the maxilla may also be lost. Also, functional losses can be severe as a result of such surgical resections. The loss of the oral cavity may lead to the malfunctions in saliva control, swallowing, chewing and speaking [12]. Along with the cosmetic losses, thefunctional losses create serious psychological trauma in the patients as well as their relatives. However, because of the development of materials and techniques in recent years, patients with such kinds of defects can be successfully rehabilitated through prosthetic restorations. In the cases of large defects, the monoblock prosthesis is preferred for the restoration, including the orbital-nasal and cheek regions, not including the oral cavity and lip. Moreover, for the complex reconstructions of extensive defects in the midfacial region, microvascular free tissue transfer in combination with extraoral implants and craniofacial prosthetic work provides reasonable functional as well as aesthetic outcomes and certainly improves the quality of life. For the retention of the prostheses, the usage of cranio-facial and zygomatic implants is very important [13]. The use of tissue undercuts and adhesives is not possible for the retention of large prostheses. In order to get the best results, surface structures and contours of the prosthesis should be in accordance with that of the patient's skin as the compliance of prosthetic surface with the patient's skin is very crucial. Intra-oral and extraoral prostheses are generally used in combination for midfacial defects. In cases of mid facial defects, the aesthetic desires are not 
as important as the requirement for filling the defect. In such cases, sensitive retentioners are used for only retention indication without any aesthetic purpose in treatments of intra-oral defects [14].

\section{DISCUSSION}

Choice of rehabilitation of the maxillofacial defects by surgical or prosthetic methods depends upon the patient's desire as well as size and cause of the defects [15]. The young patients usually wish their facial region to be treated with their own tissues rather than prostheses while the older patients usually desire to be treated by using prostheses. In general, the small maxillofacial defects are treated by the surgical methods, while the larger defects are treated by the prosthetic restorations to give a more natural appearance.

\section{CONCLUSION}

The maxillofacial prostheses should restore the lost tissues which cannot be rehabilitated by the plastic surgery, including its shape, color and texture, which should be in accord with near tissues. The prostheses must not be noticeable to the society because a noticeable prosthesis enhances the anxiety of patient as well as it does not allow the desired social adaptation to occur.

\section{REFERENCES}

1. Andreopoulos, A. G., \& Theophanides, T. (1993). Design and properties of maxillofacial prosthetic materials, Med Device Technol, 4, 20-5.

2. Hanson, M. D., Shipman, B., Blomfield, J. V., \& Janus, C. E. (1983). Commercial cosmetics and their role in the coloring of facial prostheses. $J$ of Prosthet Dent, 50, 818-820.

3. Lewis, D. H. \& Castleberry, D. J. (1980). An assessment of recent advances in external maxillofacial materials. J of Prosthet Dent, 43, 426- 32.

4. Murat, S., Gürbüz, A., Günay, Y. \& Oruç, A. S. (2011). Aesthetic approaches in maxillofacial prosthesis. J Dental Sci-Special Topics, 2, 97-114.
5. Guttal, S. S., Patil, N. P., Nadiger, R. K., \& Kulkami, R. (2008). A study on reproduction silicone shade guide for maxillofacial prostheses matching Indian skin color. Indian J Dent Res, 19, 191-5.

6. Okubo, S. R., Kanawati, A., Richards, M. W., \& Childress, S. (1998). Evaluation of visual and instrument shade matching. J of Prosthet Dent, 80, 642-8.

7. Rahn, A. O., \& Boucher, L. J. (1970). Maxillofacial prosthetics principles and consepts. Philedalphia; W.B. Saunders Company.

8. Sweeney, W. T., Fischer, T. E., Castleberry, D. J., \& Cowperthwaite G. F. (1972). Evaluation of improved maxillofacial prosthetic materials. $J$ of Prosthet Dent, 27, 297-305.

9. Zyl, I. V. \& Geissberger, M. (2001). Simulated shape design. JADA, 132, 1105-1109.

10. Aydınatay, K., Korkmaz, T., Coşkun, A., Yılmaz, C., \& Yaluğ, S. (2004). Analysis of heat caused change colored and noncolored maxillofacial elastomers. J of Dental Faculty of Cumhuriyet University, 7, 11-19.

11. Craig, R. G., Koran, A., Yu, R., \& Spencer, J. (1978). Color stability of elastomers for maxillofacial appliances J Dent Res, 57, 866-871.

12. Eleni, P. N., Katsavou, I., Krokida, M. K. \& Polyzois, G. L. (2008). Color Stability of facial silicone prosthetic elastomers after artificial weathering. Dent Res J, 5, 71-79.

13. Haddad, F. M., Goiato, C. M., Micheline Dos Santos, D., Moreno, A., Filipe Deealmeida, N. \& Alves Pesqueira, A. (2011). Color stability of maxillofacial silicone with nanoparticle pigment and opacifier submitted to disinfection and artificial aging. J Biomed Opt, 16, 095004.

14. Haug, S. P., Andres, C. J., \& Moore, B. K. (1999). Color stability and colorant effect on maxillofacial elastomers. Part III: weathering effect on color. J of Prosthet Dent, 81, 431-8.

15. Kiat-Amnuay, S., Johnston, D. A., Powers, J. M. \& Jacob, R. F. (2005). Color stability of dry earth pigmented maxillofacial silicone a-2186 subjected to microwave energy exposur. J Prosthodont, 14, 91-96. 